\title{
Verbraucherschutz auf dem Sozialmarkt
}

\author{
Die Nutzung marktwirtschaftlicher Instrumente setzt bei den \\ Leistungsberechtigten Kompetenzen voraus, \\ über die sie möglicherweise gar nicht verfügen
}

Monika Burmester

Prof. Dr. Monika Burmester lehrt seit Herbst 2008 Ökonomie des Sozial- und Gesundheitswesens mit dem

Schwerpunkt Betriebswirtschaftslehre an der Evangelischen Fachhochschule Rheinland-Westfalen-Lippe. Zuvor war sie Referentin im Diakonischen Werk der EKD mit Zuständigkeit u. a. für die Themen SGB II, Sozialberichterstattung, Schuldnerberatung.

E-Mail burmester@efh-bochum.de
Auf dem Sozialmarkt ändert die Verwendung des Verbraucherbegriffs nichts daran, dass es sich bei dem betrachteten Personenkreis um Menschen handelt, die gesetzliche Ansprüche an die Sozialversicherung oder die staatliche Gemeinschaft haben.

Verbraucherschutz dient dem »Schutz des privaten Endverbrauchers vor defekten oder gefährlichen Produkten, unlauteren Vertriebsmethoden, unlauteren Geschäftsbedingungen, überhöhten Preisen und Überschuldung «(Hippel 2001: 16). Bezogen auf Konsumenten von sozialen und gesundheitlichen Dienstleistungen könnte Verbraucherschutz entsprechend der Schutz vor unsachgemäßer Behandlung durch Dienstleister, vor gesundheitsschädigender oder die Würde missachtende Behandlung, vor falscher Beratung, vor rechtlich fragwürdigen oder nur einfach intransparenten Verträgen und selbstverständlich vor überhöhten Preisen für Dienst- und Sachleistungen bedeuten.

$\mathrm{Zu}$ fragen ist, ob im Sozial- und Gesundheitswesen institutionelle Arrangements gelten, die für die klassische Form des Verbraucherschutzes keine Grundlage bilden oder aber, ob die Forcierung betriebswirtschaftlicher und marktwirtschaftlicher Instrumente in diesem Bereich einen stärkeren oder auch anderen Schutz der Konsumenten erforderlich macht.

Verbraucherschutz ist in der Marktwirtschaft notwendig, weil Endverbraucher und Anbieter bzw. Produzenten entgegengesetzte Interessen verfolgen, keine annähernd gleich starken Marktpartner sind und weil sie über unterschiedliche Informationen über die gehandelten Produkte verfügen. Auf den Konsumgütermärkten treten sich Anbieter und Nachfrager gegenüber, die lediglich formal gleich sind. Faktisch sind die Möglichkeiten beider Parteien, das Marktergebnis zu ihren Gunsten zu beeinflussen, sehr ungleich verteilt.
Diese Diagnose steht in gewissem Widerspruch zu dem, was Ökonomen und zunehmend auch Sozialpolitiker als Vorteil marktmäßiger Koordinationsmechanismen propagieren. Danach bestimmen eigentlich die Konsumenten mit ihrer Nachfrage darüber, welche Leistungen in einer Gesellschaft bereitgestellt werden. »Die Souveränität des Konsumenten markiert einen Grundpfeiler einer freiheitlichen Wirtschaftsordnung." (Enste/Flüter-Hoffmann 2008: 5). Mit dem Eintreten für die Interessen der Konsumenten macht sich der Verbraucherschutz für die Konsumentensouveränität stark. Dazu bedarf es gut informierter Verbraucher, denn: Unternehmen können überhöhte Preise durchsetzen, weil Verbraucher nicht wissen, wo es das gleiche Produkt günstiger gibt. Sie können schlechte Qualität liefern, weil Konsumenten die Qualität nicht wirklich beurteilen können. Das gilt sowohl für Sachgüter als auch für Dienstleistungen.

Mit Verbraucherschutz wird unternehmerischem Handeln eine Grenze gesetzt, um damit die dem Marktverhältnis immanente Asymmetrie zwischen Produzenten und Verbrauchern zugunsten letzterer etwas zu korrigieren. Indem Verbraucherschutz für mehr Markttransparenz sorgen soll und durch gesetzliche Vorgaben Qualitätsstandards festlegt bzw. Gefährdungspotenziale zu vermeiden versucht, ist den Verbrauchern erst die Grundlage für eine gewisse Entscheidungsfreiheit eröffnet: Auf bestimmte Eigenschaften von Gütern können sich Verbraucher verlassen. Dass selbst diese Bedingung nicht immer gilt, machen die ständig wiederkehrenden Lebensmittelund sonstige Skandale deutlich. Gleichwohl nehmen viele Unternehmen die Produktqualität sehr ernst und nutzen sie für ihre Marktstrategie. Qualitätsmanagement ist ein wichtiges Instrument hierfür.

Letztlich bezieht sich der Schutz von Verbrauchern immer auch auf die finanzielle Ebene: Verbraucher geben ihr Geld 
für eine bestimmte erwartete Qualität aus, und sie möchten möglichst wenig ihres knappen Geldes dafür hergeben. Verbraucherschutz arbeitet sich daher regelmäßig an finanziellen Fragen ab: Sind Produkte überteuert, weil der Anbieterwettbewerb nicht richtig funktioniert? Sind die teuersten Produkte immer auch die besten? Werden Konsumenten Verträge aufgedrängt, durch die sie übervorteilt werden? Und so weiter ...

Für Leistungsnehmer auf dem Sozialmarkt spielt dieser finanzielle Aspekt in vielen Fällen keine oder keine größere Rolle. Im Sozial- und Gesundheitswesen haben die Leistungsnehmer oft gar keinen Einfluss auf die Preisgestaltung und die Definition von Leistungsinhalten. Viel- individuelle Zahlungsbereitschaft. Voraussetzung für den Leistungsbezug ist vielmehr die Anspruchsberechtigung. Sie wird vom Leistungsträger gewährt, der die Kosten der Leistungen vollständig oder zu einem Teil trägt.

Verbraucherschutz zielt auf Konsumentensouveränität. Sozialleistungen in Form persönlicher Dienstleistungen gelten ökonomisch als Eingriffe in die Konsumentensouveränität. Sie werden subventioniert, weil von diesen Gütern mehr konsumiert werden soll, als es ohne die staatliche Unterstützung der Fall wäre. Diese Form staatlicher Eingriffe wird von Vertretern der reinen Marktlehre durchaus kritisch gesehen, denn: "Welcher Konsum richtig oder falsch ist, hängt

\section{"Sozialleistungen in Form persönlicher Dienstleistungen gelten ökonomisch als Eingriffe in die Konsumentensouveränität«}

fach sind sie über die Kosten der von ihnen in Anspruch genommenen Leistungen nicht einmal informiert. Dies gilt allerdings nicht für alle Leistungen, die auf dem Gesundheits- und Pflegemarkt gehandelt werden. Im Gesundheitsbereich werden Patienten zunehmend Leistungen angeboten, die aus dem Katalog der gesetzlichen Krankenkassen gestrichen oder gar nicht erst aufgenommen wurden. Einige dieser Leistungen wurden bereits unter dem Aspekt des Verbraucherschutzes untersucht.

\section{Der Verbraucher auf dem Sozialmarkt}

Der Sozialmarkt unterliegt besonderen Funktionsmechanismen. Der Zugang zu den auf dem Markt "gehandelten « sozialen Dienstleistungen ist anders geregelt als auf dem Markt für gewerbliche Güter. Um als Nachfrager für eine persönliche Dienstleistung auf dem Sozialmarkt auftreten zu können, zählt in der Regel nicht oder zumindest nicht ausschließlich die letztlich von der Einschätzung jedes Einzelnen selber ab. « (Enste/Flüter-Hoffmann 2008: 5) Werden staatliche Eingriffe im Einzelfall als unvermeidbar angesehen, dann gilt die Subjektförderung als geeignetes Instrument. In der aktuellen sozialpolitischen Diskussion werden u. a. Gutscheine als interessante Instrumente dargestellt. Gutscheine statten die Nachfrager mit Ansprüchen aus, die sie bei einem Anbieter ihrer Wahl geltend machen können. Das Argument für Gutscheine: Damit »wird mehr auf marktwirtschaftliche Mechanismen vertraut als auf die Richtigkeit staatlicher Planungen hinsichtlich Kosten und Leistungen." (Enste/Flüter-Hoffmann 2008: 6)

Dieser Gedanke hat eine gewisse theoretische Plausibilität, er ignoriert aber die institutionellen Gegebenheiten. Gutscheine ermöglichen es den Leistungsnehmern nicht, direkt auf Kosten und Leistungen der Anbieter Einfluss zu nehmen. Art und Umfang der Leistung, Qualitätsstandards, Personalschlüssel und die Vergütung der Leistungen werden in der Regel zwischen Leistungsträgern und Einrich- tungen ausgehandelt. Damit kommt ein wesentliches Element des Verbraucherschutzes - die Einflussnahme auf das Preis-Leistungs-Verhältnis - im Sozialund Gesundheitswesen nicht zum Tragen. Den Leistungsnehmern bleibt mit dem Gutschein die Möglichkeit und Notwendigkeit zur Ausübung des Wunschund Wahlrechts.

\section{Gutscheine - wessen Macht wird gestärkt?}

Gutscheine sind im deutschen Sozialsystem nicht neu. Das viel beachtete Hamburger Kita-Gutschein-System existiert seit fünf Jahren und ebenso lange gibt es Bildungsgutscheine als Instrument der Arbeitsmarktpolitik. Diese Instrumente wurden von Beginn an unter dem Gesichtspunkt der Berücksichtigung der Interessen der Leistungsberechtigten kritisch beurteilt. Dem Instrument Gutschein ist immanent, dass Leistungsträger damit sehr gut die Nachfrage steuern können. Solch selektive Wirkungen von Gutscheinen durch die ausgebende Institution sind bekannt, akzeptiert und vielfach sogar gewünscht. So kommen in Hamburg bevorzugt Erwerbstätige in den Genuss verlängerter Kinderbetreuung, was tendenziell zulasten von Kindern aus Stadtteilen mit sozialen Problemlagen gegangen ist (vgl. Bange/Arlt 2008: 410 f.). Das knappe Gut »Betreuungskapazität « wird mit Gutscheinen auf andere Nachfragergruppen verteilt.

Selektivität ist ein nicht gerade unwichtiger Aspekt bei Gutscheinen. Hier ist einerseits die Frage der Vergabe von Gutscheinen angesprochen: Welcher Personenkreis wird bei der Vergabe von Gutscheinen besonders berücksichtigt? Es geht aber auch um die Frage, welche Personengruppen das Instrument Gutschein besonders gut nutzen (können). Eine Untersuchung zu Bildungsgutscheinen in der Arbeitsförderung identifiziert beide Selektionseffekte. Zum einen haben Personen ohne Schul- und/oder Berufsausbildungsabschluss wesentlich seltener Bildungsgutscheine erhalten als höher qualifizierte Arbeitslose, zum anderen ist die Wahrscheinlichkeit, die Gutscheine einzulösen bei gering qualifizierten Arbeitslosen signifikant niedriger (vgl. Kruppe 2008: 29). Die geringere Inanspruchnahme von Gutscheinen durch Menschen mit 
besonderem Förderungsbedarf kann durchaus darauf zurückgeführt werden, dass sich die Leistungsberechtigten von diesem Instrument überfordert fühlen. »Was unter der Zielsetzung , Wahlfreiheit $<$ angetreten ist, ist gerade für diejenigen, die auf Beratung und Qualifizierung angewiesen sind, schlicht eine Überforderung. « (Kühnlein/Klein 2003: 8). Die Nutzung marktwirtschaftlicher Instrumente setzt bei den Leistungsberechtigten Kompetenzen voraus, über die sie möglicherweise nicht verfügen. Gerade wenn es um Menschen mit Unterstützungsbedarfen geht, ist jeweils zu klären, ob sie überhaupt die Rolle souveräner Konsumenten ausfüllen können. Ist Verbraucherschutz in diesen Fällen also die Voraussetzung für gute Lösungen oder sind NichtMarktstrategien vorzuziehen?

\section{Neuausrichtung der Anbieter notwendig}

»Das Gutscheinsystem fördert die Konkurrenz der Anbieter zugunsten des Kunden und stärkt dessen Nachfragemacht. « (BMFSFJ 2008: 5) Die Verwendung des Kundenbegriffs ändert nichts daran, dass es sich bei dem betrachteten Personenkreis um Leistungsberechtigte handelt. Wer welche Leistungen erhält, entscheidet sich nicht auf dem Markt aufgrund individueller Zahlungsbereitschaft. Mit Gutscheinen erhalten Leistungsberechtigte aber Ansprüche, die für Einrichtungen Kaufkraft darstellen. Mit diesem Instrument ändern sich die Marktbedingungen für die Einrichtungen insbesondere dann, wenn die Gutscheine auf andere Kundengruppen fokussieren. Die Einrichtungen und Dienste müssen sich stärker als in früheren Strukturen um die Leistungsnehmer bemühen und zwar vor allem um diejenigen, die mit den Gutscheinen ausgestattet sind. Sie müssen gegebenenfalls ihre Leistungsangebote modifizieren, um neue Kunden zu gewinnen.

Wirklich neu sind diese Bedingungen für die Sozialunternehmen allerdings nicht. Krankenhäuser konkurrieren um Patienten, Pflegeheime um Pflegebedürftige usw. Die Konkurrenz um die Kunden findet nur dann nicht statt, wenn Einrichtungen gut belegt sind. Entsprechend der Marktsituation ist auch das Instrumentarium für die Berücksichtigung der Kundeninteressen in den verschiedenen Berei- chen des Sozial- und Gesundheitswesens unterschiedlich entwickelt. Eine stärkere Orientierung an den Kundeninteressen wäre in allen Bereichen wünschenswert. Dies ist ein wichtiger Baustein für den Schutz der Konsumenten sozialer Dienstleistungen.

\section{Literatur}

Bange, D./Arlt, S. (2008): Das Hamburger »Kita-Gutschein-System «. Eine erste Bilanz, in: NDV 10, S. 409-414.

BMFSFJ (2008): Gutscheine. Gezielte Förderung für Familien. Monitor Familienforschung Ausgabe 12. Internet http://www.bmfsfj.de/bmfsfj/generktor/RedaktionBMFSFJ/Abteilung2/New sletter/Monitor-Familienforschung/ 2008-01/monitor-familienforschung12,property=pdf, bereich $=, \mathrm{rwb}=$ true.pdf [Zugriff: 12.12.2008]

Enste/Flüter-Hoffmann 2008: Gutscheine als Instrument einer effizienten und effektiven Sozialpolitik sowie betrieblichen Personalpolitik. Bestandsaufnahme und Analyse von betrieblichem Nutzen und volkswirtschaftlichen Effekten verschiedener Gutscheinsysteme. IW Köln. Internet http://www.iwkoeln.de/Portals/0/pdf/pressemappe/ 2008/pma_180108_Endbericht\%20Gutscheine.pdf [Zugriff: 12.12.2008]

Hippel, Eike v. (2001): Präventiver Verbraucherschutz: Vorbeugen ist besser als Heilen, in: Aus Politik und Zeitgeschichte. B 24, S. 16-22.

Kruppe, T. (2008): Selektivität bei der Einlösung von Bildungsgutscheinen. IAB-Discussion Paper 17/2008.

Kühnlein, G./Klein, B. (2003): Bildungsgutscheine - mehr Eigenverantwortung, mehr Markt, mehr Effizienz? Hans-Böckler-Stiftung. Arbeitspapier 74.
Berufsbegleitender

Masterstudiengang

\section{Sozialinformatik}

Erstmals in Deutschland:

Qualifizierte Hochschul-Ausbil-

dung zum IT-Experten für die

Sozialwirtschaft

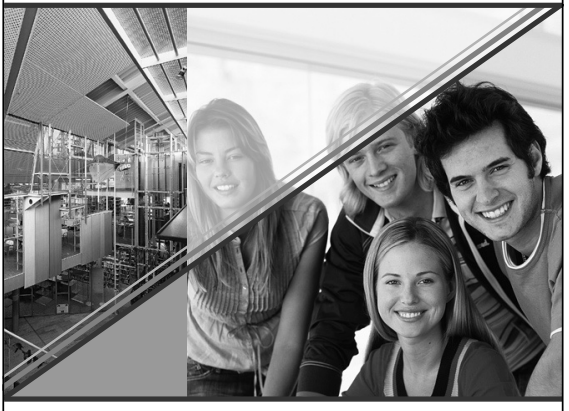

- Dauer: 4 Semester, Blockveranstaltungen + E-Learning

- Start: Oktober 2009

- Bewerbungsschluss: 31. 7. 2009

- Abschluss: Master of Arts

- Studiengangsleitung:

Prof. Helmut Kreidenweis

Gerne senden wir Ihnen eine ausführliche Studiengangsbeschreibung und weitere Informationen zu.

Kath. Universität Eichstätt-Ingolstadt Fakultät für Soziale Arbeit Ostenstr. 26 85071 Eichstätt sozialinformatik-master@ku-eichstaett.de sozialinformatik.de/master

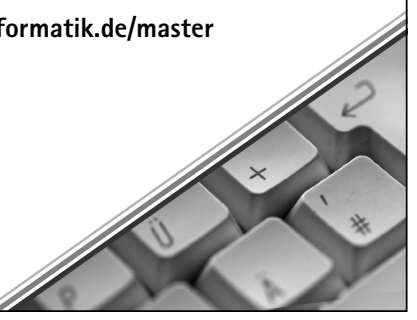

